\title{
TMS over M1 Reveals Expression and Selective Suppression of Conflicting Action Impulses
}

\author{
A. Dilene van Campen ${ }^{1}$, Max C. Keuken ${ }^{1,2}$, \\ Wery P. M. van den Wildenberg ${ }^{1}$, and K. Richard Ridderinkhof ${ }^{1}$
}

\begin{abstract}
Goal-directed action control comes into play when selecting between competing action alternatives. Response capture reflects the susceptibility of the motor system to incitement by taskirrelevant action impulses; the subsequent selective suppression of incorrect action impulses aims to counteract response capture and facilitate the desired response. The goal of this experiment was to clarify physiological mechanisms of response capture and suppression of action impulses during conflict at the level of the motor system. We administered single-pulse TMS at various intervals preceding speeded choice responses. The correct response side was designated by stimulus color, whereas stimulus location (which could match or conflict with response side) was to be ignored. TMS pulses triggered motor evoked potential and silent period, providing sensitive indices of cortico-spinal excitation and
\end{abstract}

inhibition. Motor evoked potential data showed the typical progressive increase in cortico-spinal motor excitability leading up to the imminent (correct) response, which started earlier on nonconflict than on conflict trials. On conflict trials, the irrelevant stimulus location captured the incorrect response, as expressed by an early and transient rise in excitability. Silent period data showed that, already early during the response process, inhibition of the incorrect response was stronger for conflict than for nonconflict trials. Furthermore, inhibition decreased over time for nonconflict trials facilitating the imminent correct response while maintaining higher levels of inhibition on conflict trials. In conclusion, dynamic patterns of cortico-spinal excitability provide unique physiological evidence for the expression and selective suppression of action impulses captured by competing action alternatives.

\section{INTRODUCTION}

Cognitive control facilitates goal-directed behavior and comes into play when selecting between competing action alternatives (Egner \& Hirsch, 2005). Conflict paradigms, such as the Flanker, Stroop, and Simon tasks, present both task-relevant and task-irrelevant stimulus information, giving rise to conflict between simultaneously activated action alternatives (MacLeod, 1991; Eriksen \& Eriksen, 1974; Simon, 1969). Traditionally, the conflict effect is expressed by the difference in RT between conflict trials that afford two competing actions and nonconflict trials. Here, we use single-pulse TMS to uncover the cortico-spinal dynamics of the expression and subsequent suppression of conflicting action impulses. In conflict tasks, response capture reflects the susceptibility of the action system to incitement by action impulses that are inadvertently triggered by task-irrelevant stimulus attributes. The subsequent selective suppression of these incorrect action impulses aims to counteract interference and facilitate the designated response (Kornblum, Hasbroucq, \& Osman, 1990).

The Dual Process Activation-Suppression (DPAS) model dissociates between these two temporally distinct

${ }^{1}$ University of Amsterdam, The Netherlands, ${ }^{2}$ Max Planck Institute for Human Cognitive and Brain Sciences, Leipzig, Germany

processes and predicts that their dynamics are expressed behaviorally in RT distributions (Ridderinkhof, 2002). Whereas response capture manifests in the incidence of fast errors on conflict trials, its subsequent suppression is evident in the RT distribution as a salient reduction of the conflict effect in slow compared with fast responses. Amassed evidence supports these predictions, with RT distribution analyses revealing variations in response capture and inhibitory proficiency (e.g., as a function of Parkinson's disease severity). These variations remain largely concealed when analyses are confined to mean RTs (for a review, see van den Wildenberg, Wylie, et al., 2010). The pre-SMA and right inferior frontal cortex (rIFC) have been associated with the selection of appropriate actions (in particular, when facing competing alternatives) and the suppression of inappropriate actions, respectively (for a review, see Ridderinkhof, Forstmann, Wylie, Burle, \& van den Wildenberg, 2011). Proneness to response capture, reflected by the incidence of fast errors on conflict trials, covaried with increased activation of the pre-SMA during conflict trials, such that individuals who showed more impulse capture also showed greater pre-SMA activation, suggesting that pre-SMA was engaged more strongly to select the correct response in the face of action conflict. The proficiency of suppressing this response capture, as indicated by a pronounced reduction of the interference 
effect, covaries with activation of the rIFC (Forstmann, Jahfari, et al., 2008; Forstmann, van den Wildenberg, \& Ridderinkhof, 2008).

TMS over the motor system provides an effective tool for studying the temporal dynamics of action control (Neubert, Mars, Buch, Olivier, \& Rushworth, 2010; Taylor, Nobre, \& Rushworth, 2007; Burle, Bonnet, Vidal, Possamaï, \& Hasbroucq, 2002). Here, we use TMS to unveil the temporal interplay between the expression and suppression of action impulses during conflict. TMS over the primary motor cortex (M1) yields two distinct measures in the electromyogram (EMG) recorded from effector muscles. The amplitude of the motor evoked potential (MEP) indexes excitability of the cortico-spinal tract, whereas the duration of the late part of the silent period (SP) reflects the involvement of inhibitory neural circuits intrinsic to M1 (Terao \& Ugawa, 2002). The buildup of response activation is reflected by increasing MEP amplitudes as action preparation unfolds following stimulus presentation. Previous TMS studies linked MEP amplitudes to the dynamic activation and inhibition of overt responses. For example, in stop tasks, ongoing motor actions should be cancelled upon presentation of a stop signal. On stop trials, the buildup of response activation is curtailed and reversed preceding the successful inhibition of the imminent response, a pattern not observed when attempted inhibition fails (van den Wildenberg, Burle, et al., 2010). When comparing conflicting and nonconflicting Simon trials to neutral trials, increased cortico-spinal excitability reflects the priming of the spatially corresponding response hand. MEP amplitudes were magnified if stimulus location primed the correct response hand (Stürmer, Siggelkow, Dengler, \& Leuthold, 2000). However, the temporal dynamics and differentiation between activation and inhibition components remain to be explored.

The goal of this experiment was to clarify physiological mechanisms of response capture and suppression of action impulses during conflict at the level of the motor system. Within the action-conflict paradigm, the DPAS model predicts an early capture of the incorrect response (increased MEP), followed by selective suppression of these activation tendencies (prolonged SP duration).

\section{METHODS}

\section{Participants}

This study included 10 participants (six men, $M$ age $=$ 27.5 years, $S D=5.6$ years). All participants were screened according to the international screening guidelines for TMS research (Rossi, Hallett, Rossini, \& PascualLeone, 2009) and provided written informed consent before participation. All procedures were approved by the local ethics committee and complied with relevant laws, institutional guidelines, and the international guidelines for TMS procedures (Rossi et al., 2009).

\section{Apparatus}

Task

A two-color (green and blue) Simon task was shown on a computer screen (17-in. digital display). The computer screen was placed in front of the participant at a distance of approximately $90 \mathrm{~cm}$, and the stimuli appeared at eye level. Each trial started with a fixation cross $(0.5 \times 0.5 \mathrm{~cm})$ in the middle of a white screen, which disappeared at the end of the trial (maximum duration of $2500 \mathrm{msec}$ ). A colored circle $(2 \mathrm{~cm}$ in diameter) appeared on either the right or left side of the fixation cross (edge-to-edge distance between fixation cross and circle was $0.6 \mathrm{~cm}$ ) and disappeared after a response was made (maximum duration $=1500$ msec; see Figure 1A). Participants were instructed to issue a left- or right-hand button press as quickly and accurately as possible according to the color of the circle. Half of the participants responded right to blue circles and left to green circles; this mapping was reversed for the other half.

Circles could appear left or right of fixation. Although task irrelevant, the stimulus location inadvertently triggers an involuntary action impulse of the ipsilateral hand; for example, circles presented to the right side activate the right-hand response, irrespective of color (see Figure 1A). On the basis of the correspondence between the location of the stimulus and the correct response hand on a given trial, the action selection according to color is either facilitated or hampered by the involuntary action impulse that is triggered by the position of the circle. Responses are typically fast and accurate on corresponding (CR) trials, in which the relevant and irrelevant aspects converge to the same hand (i.e., when a green circle requiring a left-hand response is presented to the left of fixation). Alternatively, on noncorresponding (NCR) trials, RT is delayed and error levels are typically elevated because the two processing streams activate conflicting response tendencies (e.g., when a green circle that should be responded to by the left hand is presented to the right of fixation). Within a block of 112 trials, an equal amount of CR and NCR trials were randomly distributed. After each block, a feedback screen was presented with mean RT and accuracy of that block.

\section{Force Recordings}

Responses were recorded with force buttons mounted onto a grip; responses were given with the tip of the thumbs while holding a grip in each hand (see van den Wildenberg, Burle, et al., 2010). The participant initiated each trial by generating tonic force levels, yielding tonic background activity in the EMG, to distinguish the SP. Tonic force had to be maintained for $1000 \mathrm{msec}$ within a specific force window (2-5 N) before the fixation cross would appear on the screen, and the trial was initiated. The upper and lower force boundaries of the force window were fixed. An overt response (RT) was marked if 


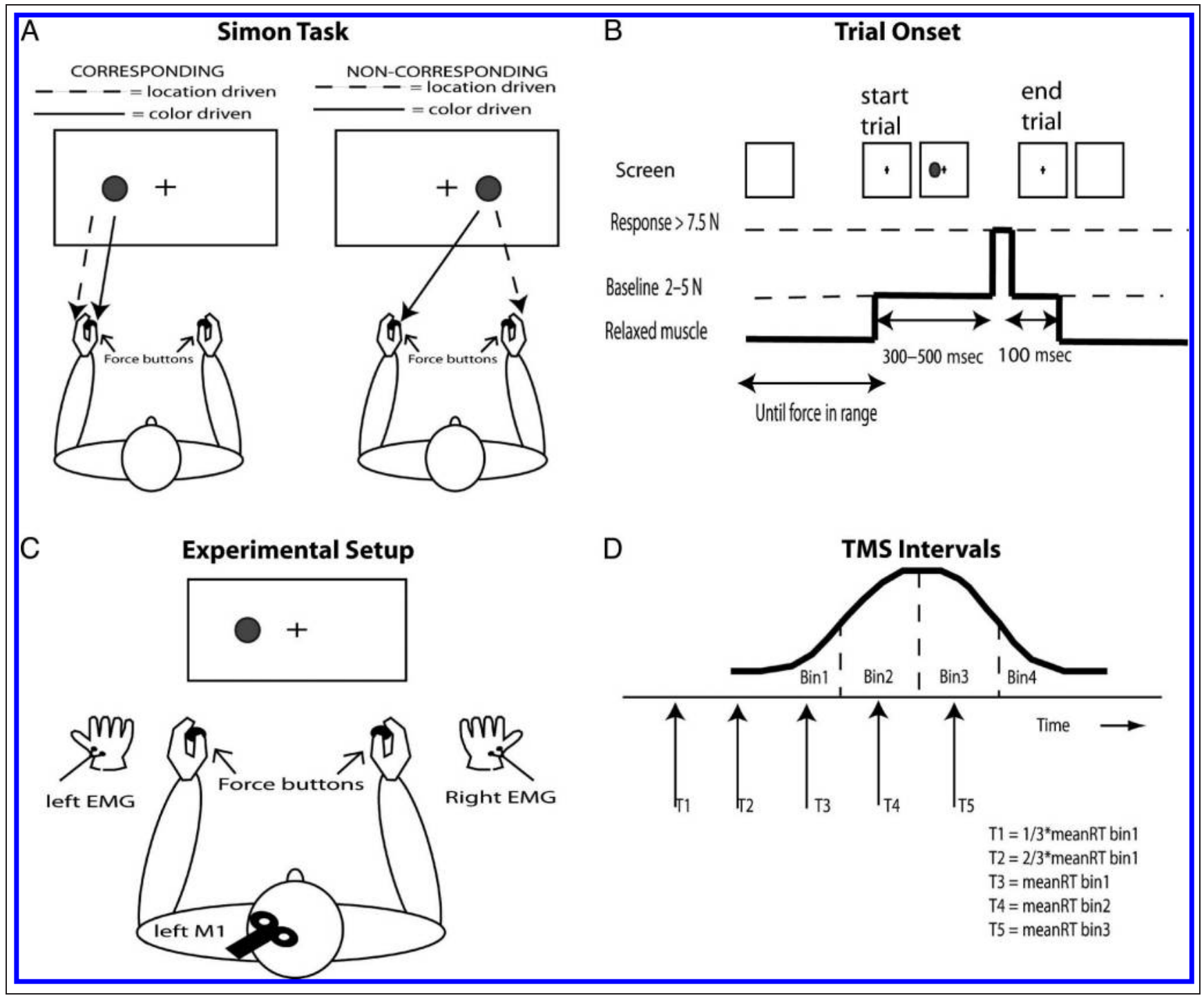

Figure 1. Task setup. (A) Simon task. Colored circles appear either on the left or right side of the screen. On CR trials, both the location and the color of the circle drive the correct response hand. On NCR trials, the goal-directed correct response conflicts with the response activation driven by the task-irrelevant stimulus location. (B) Trial sequence. To start the trial, the participant generates a tonic force between 2 and $5 \mathrm{~N}$. This procedure yields background EMG activity from the APB muscles in the hand necessary for SP recording. The force criterion for RT was set at 7.5 N. (C) Setup of the TMS coil over the left M1. EMG activity from both the left and right APB is measured. (D) RT distribution is established for each individual, and individual timing intervals are calculated based on the individual RT distribution. During a trial, potentially only one TMS pulse is given.

the force exceeded a threshold of $7.5 \mathrm{~N}$ (see Figure 1B). A feedback environment was used to learn the sensitivity of the force window. Above the screen, an LED system indicated the amount of force applied to the force buttons. The LED system visualized the applied amount of force during the first block of the first session to help the participant to learn how much force to apply to initiate the trial.

\section{EMG Recordings}

EMG activity of the right and left abductor pollicis brevis (APB) was measured using the VSRRP System (in-house built; Technical Division, Department of Psychology,
University of Amsterdam). For each muscle, two cup electrodes and a ground band were used. The sample rate was $2000 \mathrm{~Hz}$. Baseline EMG activity (necessary for precontraction activity) was monitored continuously during the experiment (see Figure 1B and C).

\section{Procedure}

The experiment consisted of two sessions on 2 separate days: The first session was a behavioral session, and in the second session, TMS was applied. Both sessions were performed using the same experimental setup. The main difference between the sessions was the application of TMS. The behavioral session consisted of four practice 
blocks and four experimental blocks. At the end of the behavioral session, participants were introduced to TMS, and test pulses were delivered to familiarize the participants with the TMS setup. The second session (with TMS) consisted of 10 blocks. This session was similar to the behavioral session but was preceded by a protocol (described below) to determine the correct location and intensity for the TMS pulse.

\section{TMS}

TMS over the left motor cortex was conducted with a Magstim System 200. Participants were seated in a chair with a head support system. This framework was used to minimize movements between the figure of eight coil and the head of the participant (see Figure 1C). Searching for the hotspot, defined as the location resulting in the largest MEP amplitude in the APB muscle of the right hand, started $2 \mathrm{~cm}$ lateral and $1 \mathrm{~cm}$ frontal to the vertex. The hotspot was marked with a skin-friendly marker to check for coil dislocation between blocks of trials. For each individual, two thresholds were established: resting and active threshold. For resting threshold, the lowest intensity was taken at which the MEP amplitude was $>50 \mu \mathrm{V}$ in 5 of 10 pulses although the muscle was in a relaxed state. For active threshold, the lowest intensity was taken at which the MEP amplitude was $>250 \mu \mathrm{V}$ measured with a slight muscle contraction (monitored online). During the TMS session, the intensity was set at $110 \%$ of active motor threshold. It was checked that the set intensity did not interfere with the response. In one case, this resulted in disturbance of the response. Here, we slightly adjusted the threshold to the point that the pulse did no longer disturb the response (see Table 1 for participant characteristics).

\section{TMS Intervals}

For each participant, five TMS intervals were defined based on individual RT distributions of CR right-hand trials assessed in the behavioral session and binned into four equal-sized segments (quartiles). The first two TMS intervals were set to one third and two thirds of the mean RT of the first bin; TMS intervals 3, 4, and 5 were set to the mean RT of bins 1, 2, and 3, respectively (see Figure 1D). To compensate for the delay between EMG onset and the overt mechanical response, $100 \mathrm{msec}$ was subtracted from the RT mean of each bin based on pilot data. The mean TMS intervals were 71, 142, 213, 264, and $314 \mathrm{msec}$, respectively, after stimulus onset (see Table 1 for individual TMS intervals). The number of trials with a specific TMS interval was the same for each block. Because of the higher chances of responding before the TMS pulse, the distribution of TMS intervals $1-5$ was $12,12,12,20$, and 32 , respectively, per interval for each trial type. Within each block, there were also 24 trials without TMS to discourage anticipation of the TMS pulse.

\section{Data Analyses}

\section{Behavioral Data}

Trials with RTs of $>1000 \mathrm{msec}$ and $<100 \mathrm{msec}$ were identified as outliers and removed from the analyses. Mean RT and accuracy levels were calculated separately for CR and NCR trials. The mean Simon interference effect was calculated as the difference between mean RT on correct NCR and CR trials. To quantify the temporal dynamics, RT distributions for CR and NCR trials were rank ordered and divided in four equal-sized bins (quartiles). For each bin, the mean RT for correct CR and NCR trials was established, and the differences between those means (Simon effect per bin) were plotted against the mean RT of each

Table 1. Individual Participant Characteristics

\begin{tabular}{lcccccccc}
\hline Participant & Age & AMT \% & Test \% & Interval 1 & Interval 2 & Interval 3 & Interval 4 & Interval 5 \\
\hline 1 & 36 & 39 & 43 & 69 & 139 & 208 & 250 & 296 \\
2 & 30 & 31 & 35 & 80 & 160 & 241 & 284 & 333 \\
3 & 20 & 35 & 39 & 63 & 126 & 190 & 236 & 326 \\
4 & 36 & 38 & 40 & 65 & 131 & 196 & 232 & 252 \\
5 & 24 & 47 & 52 & 81 & 163 & 244 & 312 & 378 \\
6 & 25 & 36 & 40 & 66 & 133 & 199 & 243 & 279 \\
7 & 22 & 48 & 52 & 65 & 131 & 196 & 232 & 258 \\
8 & 23 & 31 & 34 & 73 & 147 & 220 & 263 & 307 \\
9 & 29 & 40 & 44 & 64 & 129 & 194 & 265 & 330 \\
10 & 30 & 38 & 43 & 81 & 162 & 243 & 322 & 385 \\
\hline
\end{tabular}

Age, active motor threshold (AMT in percentage of maximum stimulator output), test intensity (test in percentage of maximum stimulator output) at $110 \%$ AMT, and individual TMS intervals (intervals 1-5). 
bin; this was graphically represented in a delta plot. Both the Simon effect within each bin (delta values) and the slopes connecting subsequent delta values were taken as dependent measures (see van den Wildenberg, Wylie, et al., 2010). For accuracy levels, percentage correct for CR and NCR trials were calculated for each bin and plotted against the mean RT of that specific bin, graphically represented in a conditional accuracy function (CAF).

\section{Physiological Data}

MEP and SP were calculated from the EMG signal for each trial using an automatic tracking system and visual inspection by two experienced raters (interrater reliability was above .80). MEP amplitude was defined as the absolute difference between the highest peak and the lowest peak within a 40-msec window after the TMS pulse. To correct for possible differences in baseline EMG levels, the EMG activity during the 100-msec interval preceding the TMS pulse was calculated for each trial separately, and MEP amplitude was divided by this baseline. The SP started at the point where the end phase of MEP crossed the averaged baseline EMG level. Reoccurrence of background EMG activation marked the end of the SP. This time point was mathematically defined as when the tangents exceeded $2 S D$ of the averaged baseline EMG level from the previous moment.

Second, all trials were visually inspected. After visual inspection, only trials with valid MEP and/or SP scores were included. Trials with a TMS pulse occurred during or after the response, trials containing double responses and trials on which the raters disagreed were excluded. Furthermore, only physiological measures of correct trials were included in the analyses. On the basis of the above criteria, the number of trials included for the analyses differed between conditions and participants. Mean number of trials per bin for left-hand responses averaged over participants were 18, 18, 17, 29, and 44 trials, respectively. For right-hand responses, fewer trials remained because of exclusion based on coinciding of the MEP or SP with the overt response $(18,18,17,19$, and 15 trials, respectively). To account for differences between participants, all physiological data were normalized to $z$ scores (Burle et al., 2002). The range of raw MEP amplitudes was $176-5483 \mu \mathrm{V}$, with a mean of $1597 \mu \mathrm{V}$ and $S D$ of $819 \mu \mathrm{V}$.

The stimulated motor cortex (left hemisphere) is either directly involved (in case of right-hand responses) or not (in case of left-hand responses). Hence, physiological measurements reflected the state of either the directly involved cortico-spinal track or the opposite (noninvolved) side.

\section{Statistical Analyses}

Univariate repeated measures ANOVAs were applied to the various dependent measures derived from behavioral data (mean RT, overall accuracy, and various measures obtained from RT distributions) and physiological measurements (MEP amplitude, SP duration). The ANOVAs included the within-subject factors session (behavioral vs. TMS) and correspondence (CR vs. NCR). For distributional analyses, the additional factors bin (bins 1-4) and slope (slopes 1-2, 2-3, and 3-4) were included. For physiological measures (MEP, SP), the additional factors hemisphere (involved in correct right-hand responses and noninvolved measured during correct left-hand responses) and TMS interval (T1-T5) were included. Four planned pairwise comparisons between the TMS intervals (T1 vs. T2, T2 vs. T3, T3 vs. T4, and T4 vs. T5) were conducted on the physiological measures to track changes over time. Exploratory analyses included the additional within-subject factor sequence (preceding trial was CR vs. NCR).

When the sphericity assumption was violated, degrees of freedom were corrected using the Greenhouse-Geisser (GG) method using SPSS 18.0. Uncorrected $d f$ s are reported for ease of reading. Pearson correlations tested the relationships between physiological and behavioral data, with alpha set at .01 to correct for multiple comparisons.

\section{RESULTS \\ Performance Data}

\section{Mean $R T$}

Mean RTs were analyzed using a two-way ANOVA with factors session and correspondence. RTs were longer on NCR trials compared with CR trials (428 msec vs. $407 \mathrm{msec}$; main effect of correspondence: $F(1,9)=28.017, p<$ .001), reflecting the typical Simon effect. RTs were longer in the behavioral session than in the TMS session ( $437 \mathrm{msec}$ vs. 398 msec; main effect of session: $F(1,9)=8.072, p=$ .019). This speeding effect associated with TMS has been reported previously (Hasbroucq, Kaneko, Akamatsu, \& Possamaï, 1997). Interaction effects failed to obtain statistical significance (Session $\times$ Correspondence, $F<1$ ), indicating that the mean Simon effect did not differ between the two sessions (23 msec during the behavioral vs. $19 \mathrm{msec}$ for the TMS session).

\section{Mean Accuracy}

Two-way ANOVA of accuracy levels, with factors session and correspondence, showed that participants made more errors in the TMS session compared with the behavioral session (7.3\% vs. 4.7\%; main effect of session: $F(1,9)=$ $10.560, p=.010)$. Responses were also less accurate on NCR compared with CR trials $(7.4 \%$ vs. $4.6 \%$ errors; main effect of correspondence: $F(1,9)=8.036, p=.020$ ), again reflecting the typical Simon effect. The interaction was not significant (Session $\times$ Correspondence: $F<1$ ), confirming that correspondence effects on errors did not differ between behavioral and TMS sessions. 


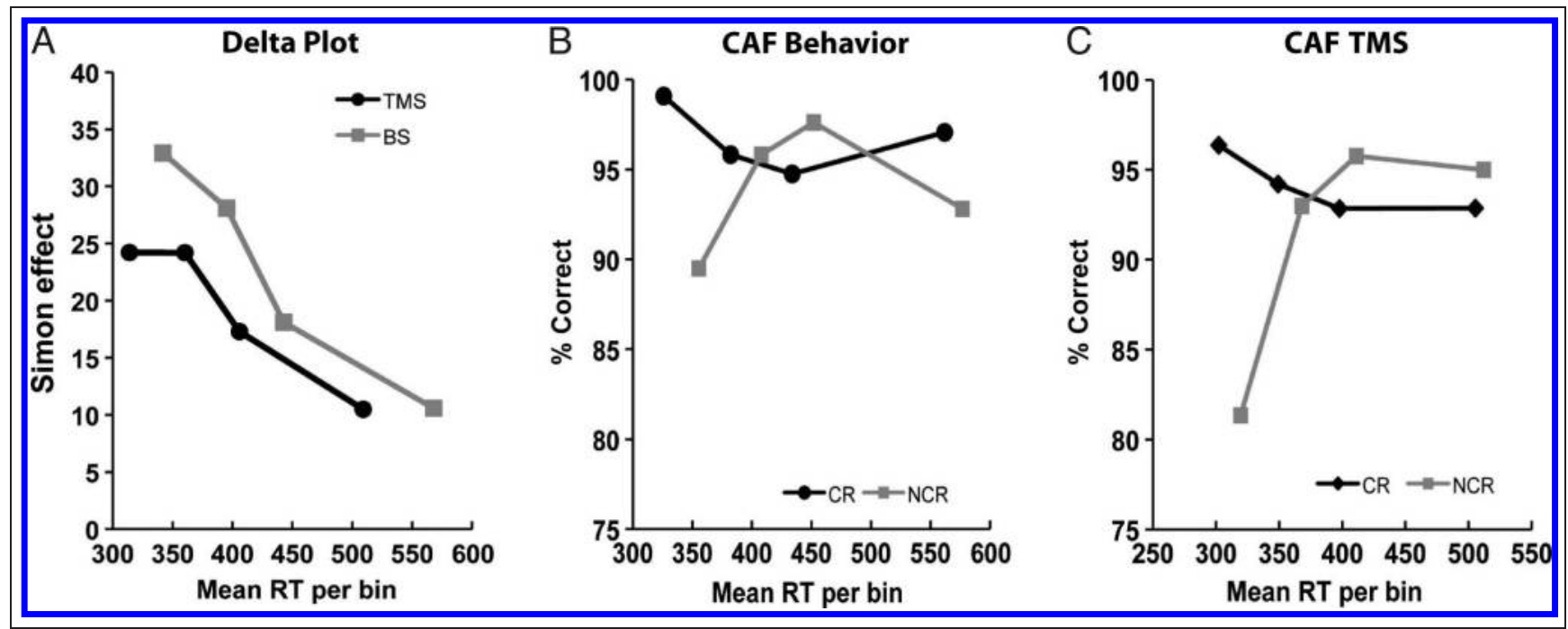

Figure 2. Delta plot and CAF. (A) Delta plot. Plotting the Simon effect within each RT bin against the mean RT per bin showed similar delta plots for the behavioral and TMS sessions. CAFs represent accuracy levels per bin plotted against the mean RT per bin. Typical patterns of high numbers of fast errors on NCR trials were obtained in the behavioral session (B) and the TMS session (C).

\section{Distributional Analyses}

Delta plots. To characterize the dynamics of activation followed by suppression of incorrect response activation, we analyzed delta bin values (i.e., the size of the Simon effect) within each bin as well as delta slopes (see Figure 2A). First, a two-way ANOVA of delta values with factors bin and session revealed that the interference effect declined over time $(29,26,17$, and $11 \mathrm{msec}$; main effect of bin: $F(3,27)=$ $6.518, p=.017$; GG corrected: $\left.\chi^{2}=17.257, \varepsilon=.473\right)$. No main effect of session $(F(1,9)<1)$ or interaction effect (Bin $\times$ Session: $F(3,27)<1)$ was found, indicating that TMS did not affect the magnitude of the interference effect. Second, a two-way ANOVA of delta slopes with factors slope and session indicated at most a trend for differences over time (slope: $F(2,18)=2.932, p=.080$ ). By and large, delta plots sloped negatively as a function of RT, a negative trend that was slightly less pronounced in the fast part of the distribution. No main effect of session $(F(1,9)<1)$ or interaction effect (Slope $\times$ Session: $F(2,18)<1)$ was obtained. In summary, TMS did not affect delta values and slope values.

CAFs. A three-way ANOVA on accuracy levels (see Figure $2 \mathrm{~B}$ and $\mathrm{C}$ ) with factors bin, session, and correspondence confirmed that participants were less accurate in the TMS session (4.7\% vs. $7.3 \%$; session: $F(1,9)=10.519, p=$ $.010)$, that participants made more errors on NCR compared with CR trials (7.5\% vs. 4.7\%; correspondence: $F(1,9)=8.061, p=.019)$, and that session and correspondence did not interact (Session $\times$ Correspondence: $F(1,9)<1)$. At most, a trend was obtained when comparing accuracy levels over bins (bin: $F(3,27)=3.682$, $p=.069 ;$ GG corrected: $\left.\chi^{2}=18.727, \varepsilon=.457\right)$; however, post hoc testing yielded no significant effects. More interesting, no interaction effect was found between session and bin $(F(3,27)=2.351)$, indicating that the dynamic pattern of accuracy was comparable across sessions. Importantly, however, an interaction effect between correspondence and bin indicated that NCR trials, but not CR trials, were associated with fast errors, replicating the typical finding of fast response capture (Correspondence $\times$ Bin: $F(3,27)=13.997, p<.001)$. A three-way interaction effect (interaction of Session $\times$ Correspondence $\times$ Bin: $F(3,27)=$ $3.527, p=.028$ ) suggested that more fast NCR errors were observed in TMS compared with behavioral sessions.

To summarize, mean RT, accuracy levels, and distributional analyses showed typical behavioral patterns that were by and large comparable between the behavioral and TMS sessions.

\section{Physiological Measures}

The MEP amplitude reflects the activation of the corticospinal track, whereas the SP duration reflects the involvement of inhibitory circuits. Figure 3 shows the cortico-spinal excitability and inhibition over time for CR and NCR trials separately for the involved (right-hand responses) and noninvolved (left-hand responses) hemispheres.

\section{MEP}

A three-way ANOVA of MEP amplitude was performed with the factors hemisphere (involved vs. noninvolved), TMS interval (T1-T5), and correspondence (see Figure 3A and B). MEP amplitudes were increased when the stimulated hemisphere was involved in the response compared with when it was noninvolved (main effect of hemisphere: $F(1,9)=18.589, p=.002)$. MEP amplitude varied over time (main effect of TMS interval: $F(4,36)=7.332$, 
$p=.006$; GHG corrected: $\left.\chi^{2}=22.508, \varepsilon=.474\right)$. The main effect of correspondence was not significant $(F(1,9)=$ $1.607, p=.237$ ). The pattern of change over time was different when the stimulated hemisphere was involved in the response compared with when it was not (interaction effect of Hemisphere $\times$ TMS interval: $F(4,36)=15.837, p<$ .001); MEP amplitudes increased as a function of time if the stimulated hemisphere was involved in the correct response (Figure 3B) but failed to show such an increase when it was not (Figure 3A). Likewise, the effect of correspondence was modulated by hemisphere: Higher MEP amplitudes were found for CR compared with NCR trials, but only when the stimulated hemisphere was involved in the correct response (interaction effect of Hemisphere $\times$ Correspondence: $F(1,9)=21.842, p=.001)$. No interaction effects were found for TMS interval $\times$ Correspondence or Hemisphere $\times$ TMS interval $\times$ Correspondence $(F<1)$.

\section{$S P$}

A three-way ANOVA of SP was performed with factors hemisphere, TMS interval, and correspondence (see
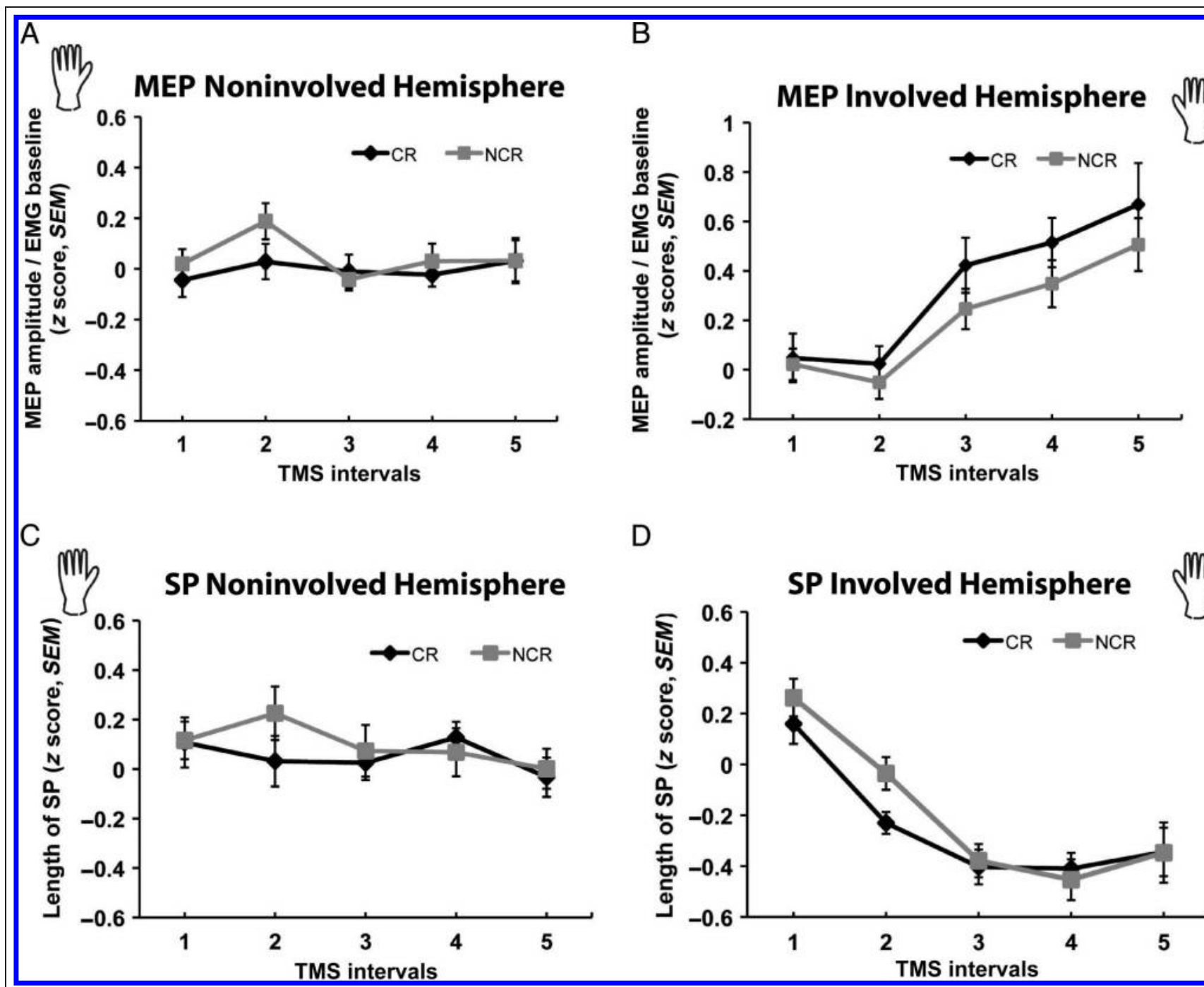

$\mathrm{D}$

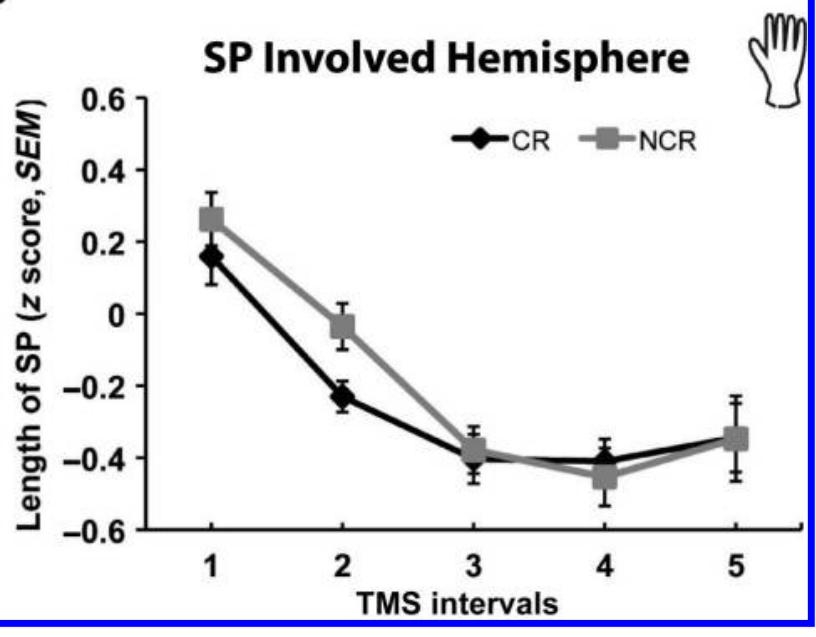

Figure 3. MEP and SP. MEP and SP of the stimulated hemisphere when it was involved in the correct response (right) and when it was not directly involved (in case of correct left-hand responses, left). Hands indicate correct response hand. In case of right-hand responses, the left hemisphere is directly involved in the correct response; in case of left-hand responses, the left hemisphere is not involved in the correct response but controls incorrect response tendencies. The top represents the MEP amplitude, which is divided by the EMG baseline and normalized to $z$ scores. The bottom represents the length of SP also expressed in $z$ scores. Error bars represent SEM. On the $x$ axis, the five TMS intervals are depicted. Mean TMS intervals over all participants are 71, 142, 213, 264, and $314 \mathrm{msec}$, respectively. (A) MEP amplitudes evoked by TMS over left M1 reflecting corticospinal activation of the incorrect (right-hand) response alternative on correct (left-hand) trials. MEP data showed an early peak around $142 \mathrm{msec}$ for NCR trials. (B) MEP amplitudes evoked by TMS over left M1 reflecting cortico-spinal activation of the correct (right-hand) trials. MEP data showed differences between CR and NCR trials and an increase over time. (C) SP duration evoked by TMS over left M1 reflecting inhibitory mechanisms of the incorrect (right-hand) response alternative on correct (left-hand) trials. SP data showed stronger inhibition of NCR trials around 142 msec. (D) SP duration evoked by TMS over left M1 reflecting inhibitory mechanisms of the correct (right-hand) trials. SP data showed a decrease of SP over time with early differences between CR and NCR trials. 
Figure $3 \mathrm{C}$ and D). A main effect of hemisphere $(F(1,9)=$ $9.720, p=.012$ ) indicated that the SP was shorter if the stimulated hemisphere was involved in the correct response. The length of the SP varied as a function of stimulation time (main effect of TMS interval: $F(4,36)=15.104, p<.001$; GHG corrected: $\left.\chi^{2}=26.918, \varepsilon=.423\right)$. No main effect of correspondence was found $(F(1,9)=2.354, p=$ .159). A gradual decline of SP duration as a function of stimulation time was observed only when the stimulated hemisphere was involved in the correct response $($ Hemisphere $\times$ TMS interval: $F(4,36)=15.513, p<$ .001 ; see Figure 3D). An interaction between correspondence and TMS interval (TMS interval $\times$ Correspondence: $F(4,36)=2.633, p=.050)$ showed that SP duration was longer for NCR than CR responses only during early stimulation intervals. No interaction effects were found between Hemisphere $\times$ Correspondence or between Hemisphere $\times$ TMS interval $\times$ Correspondence $(F<1)$.

\section{Changes over Time}

The next section summarizes the four planned comparisons $(2 \times 2$ repeated ANOVAs) with the factors correspondence and TMS interval (with [I] T1 vs. T2, [II] T2 vs. T3, [III] T3 vs. T4, and [IV] T4 vs. T5 as TMS intervals).

MEP amplitude when the stimulated hemisphere was involved in the correct response (see Figure 3B). (I) No differences in MEP amplitude were observed within the earliest (T1-T2) time intervals (correspondence: $F(1,9)<1$; TMS interval: $F(1,9)=1.143, p=.313$; Correspondence $\times$ TMS interval: $F(1,9)<1)$. (II) The MEP was larger for the T3 compared with T2 (TMS interval: $F(1,9)=14.999, p=.004)$, showing that, closer toward the response, the MEP amplitude increased. Overall, CR and NCR trials showed similar MEP amplitudes (correspondence: $F(1,9)=3.249, p=.105)$. However, the significant interaction (Correspondence $\times$ TMS interval: $F(1,9)=7.125, p=.026)$ indicated that the increase in activation over time was stronger for CR than NCR trials. (III) This pattern was consolidated between T3 and T4 with no further changes (TMS interval: $F(1,9)=1.943$, $p=.197$; correspondence: $F(1,9)=6.027, p=.036$; Correspondence $\times$ TMS interval: $F(1,9)<1$ ); MEP amplitudes remained higher for CR than NCR and increased no further. (IV) Toward the final TMS interval, the MEP amplitude difference between CR and NCR responses decreased slightly and was no longer significant (correspondence: $F(1,9)=2.116, p=.180)$, with no main effect of TMS interval $(F(1,9)=2.767, p=.131)$ or interaction (Correspondence $\times$ TMS interval: $F(1,9)<1$ ). To summarize, both CR and NCR responses showed increased activation over time, which is expressed earlier on, and remains more pronounced, for CR than NCR responses.

MEP amplitude when the stimulated hemisphere was not involved in the correct response (see Figure 3A).
MEP amplitude increased between T1 and T2 (TMS interval: $F(1,9)=6.704, p=.029)$. MEP amplitude was, as expected, higher (at trend level) for NCR than CR responses (correspondence: $F(1,9)=4.868, p=.055$ ). No interaction effect was found between Correspondence $\times$ TMS interval $(F(1,9)<1)$. (II) MEP amplitude decreased (at trend level) back to baseline between T2 and T3 (TMS interval: $F(1,9)=4.289, p=.068)$. MEP amplitude did no longer differ between CR and NCR (correspondence: $F(1,9)=1.053 p=.332$ ); the decrease from $\mathrm{T} 2$ to $\mathrm{T} 3$ was slightly more pronounced (at trend level) for NCR responses (Correspondence $\times$ TMS interval: $F(1,9)=$ $4.787, p=.056$ ). (III and IV) Pairwise comparisons for the later time intervals yielded no effects (Correspondence $x$ TMS interval 3 vs. $4, F(1,9)=2.125$; all other $F$ s $<1$ ). Overall, when the stimulated hemisphere was not involved in the correct response, early differences in activation dynamics were found indicating slightly stronger activation for NCR trials.

SP duration when the stimulated hemisphere was involved in the correct response (see Figure 3D). (I and II) The duration of the SP decreased from T1 to T2 (TMS interval: $F(1,9)=33.950, p<.001)$ and from $\mathrm{T} 2$ to $\mathrm{T} 3$ (TMS interval: $F(1,9)=15.111, p=.004)$. SP duration was shorter for CR compared with NCR responses (correspondence: $F(1,9)=22.679 / 8.332, p=.001 / .018$, for $\mathrm{T} 1-\mathrm{T} 2$ and $\mathrm{T} 2-\mathrm{T} 3$, respectively). This correspondence effect remained stable from T1 to T2 (Correspondence $\times$ TMS interval: $F(1,9)<1)$ but was nullified between T2 an T3 $(F(1,9)=5.591, p=.042)$. These patterns reflect the reduction of inhibition contralateral to the effector involved in the correct response as time progresses toward the moment of the imminent response. This disinhibition is initially less pronounced for responses to NCR stimuli. (III and IV) SP duration between T3-T4 and T4-T5 did not vary significantly as a function of any factor (all $F \mathrm{~s}<1.1$ ).

SP duration when the stimulated hemisphere was not involved in the correct response (see Figure 3C). (I) Between T1 and T2, SP duration is not influenced by correspondence $(F(1,9)=2.460, p=.151)$ or TMS interval $(F(1,9)<1)$, but a trend-wise interaction effect (Correspondence $\times$ TMS interval: $F(1,9)=4.858, p=.055)$ suggests an initial increase of inhibition for NCR but not for CR trials. (II and III) This pattern is consolidated between T2 and T3 (correspondence: $F(1,9)=5.194$, $p=.049$; TMS interval: $F(1,9)=1.671, p=.228$; Correspondence $\times$ TMS interval: $F(1,9)=1.237, p=.295)$ but then cancelled between T3 and T4 (correspondence: $F(1,9)<1$; TMS interval: $F(1,9)=1.527, p=.248$; Correspondence $\times$ TMS interval: $F(1,9)<1$ ). (IV) SP duration decreased to baseline between T4 and T5 (TMS interval: $F(1,9)=26.395, p=.001)$; no differences between CR and NCR trials or an interaction effect remained (all $F$ s $<1$ ). Overall, inhibition of the hand not involved in the correct response (but potentially involved in the incorrect 


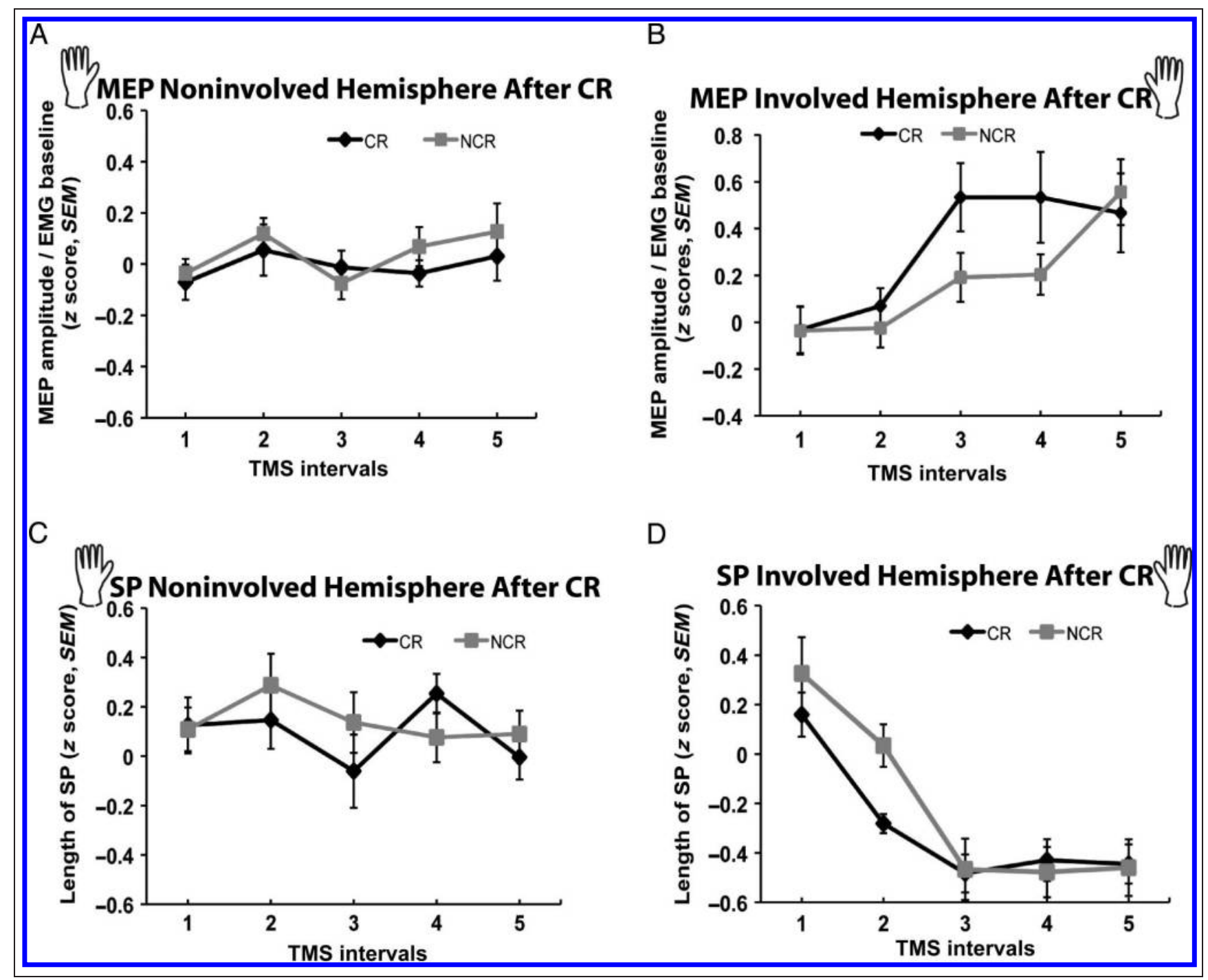

Figure 4. Sequential effects on activation and inhibition dynamics: trials following CR trials. Details and panel organization are identical to those in Figure 3.

response) is stronger for NCR trials early in time and decreased with time irrespective of correspondence.

Summary. When the stimulated hemisphere was involved in the correct response, MEP amplitude increased earlier in time (between T2 and T3) on CR trials than on NCR trials. When it was not involved in the correct response, direct activation was found early in time (T1-T2). Inhibition of the hemisphere involved in the correct response showed a gradual reduction over time (T1-T3), with initially (T1-T2) higher levels of inhibition for NCR than CR trials. The hemisphere not involved in the correct response (and occasionally involved in the incorrect response) showed an initial increase of inhibition for NCR trials (T1-T2) and an overall decrease of inhibition at late intervals (T4-T5).

\section{Exploratory Analyses of Sequential Effects}

The Simon effect is typically reduced or even reversed for trials that were preceded by NCR trials, compared with trials that were preceded by CR trials (for a review, see Egner, 2008). This effect was seen also for the present RT data in the TMS session (Simon effect: 25 msec after CR trial and 5 msec following an NCR trial), as confirmed by a two-way ANOVA (Correspondence $\times$ Sequence: $F(1,8)=$ $10.666, p=.010)$. Mainstream interpretations of this pattern suggest that, when the preceding trial was NCR, control is increased on the current trial such that the initial locationdriven activation of the incorrect response is reduced, and the suppression of that activation is strengthened (cf. Ridderinkhof, 2002). The present MEP and SP data allow for a direct test of this hypothesis. Figures 4 and 5 present the MEP and SP patterns from Figure 3, separately for trials preceded by CR trials (Figure 4) and for trials preceded by NCR trials (Figure 5). We repeated the previous analyses with the additional within-subject factor sequence. Below, we report only those patterns that deviate from those reported above in the planned comparison section. 
MEP Amplitude When the Stimulated Hemisphere Was Involved in the Correct Response (see Figures $4 B$ and 5B)

Between T1 and T2, MEP amplitude increased slightly when the preceding trial was CR but decreased slightly when the preceding trial was NCR (TMS interval $\times$ Sequence: $F(1,8)=5.746, p=.043)$. MEP amplitudes diverge between CR and NCR (larger for CR responses) when the preceding trial was $\mathrm{CR}$, but not when the preceding trial was NCR (Correspondence $\times$ Sequence, T2T3: $F(1,8)=14.452, p=.005$; and at most trend-wise for T3-T4: $F(1,8)=4.764, p=.061)$. Thus, the enhanced activation seen for $\mathrm{CR}$ responses was evident only when the preceding trial was CR. If the preceding trial was NCR, the activation pattern did not differ between CR and NCR trials.

MEP Amplitude When the Stimulated Hemisphere Was Not Involved in the Correct Response (see Figures $4 \mathrm{~A}$ and $5 \mathrm{~A}$ )

Correspondence of the preceding trial (CR vs. NCR) did not modulate the patterns of MEP amplitude measured in the hand not involved in the movement.
SP Duration When the Stimulated Hemisphere Was Involved in the Correct Response (see Figures 4D and 5D)

Correspondence of the preceding trial (CR vs. NCR) did not reliably modulate the patterns of SP durations measured from the hand involved in the movement, except that the main effect for correspondence for T2-T3 (II) was reduced to at most a trend (correspondence: $F(1,8)=4.853, p=$ $.059)$. The abolishment of cortico-spinal inhibition is initially less pronounced for NCR responses, but only when the preceding trial was CR.

SP Duration When the Stimulated Hemisphere Was Not Involved in the Correct Response (see Figures 4C and 5C)

The pattern seen in Figure 5C is slightly amplified when the preceding trial was NCR. For instance, the interaction between correspondence and TMS interval becomes more pronounced between $\mathrm{T} 1$ and $\mathrm{T} 2(F(1,7)=7.312$, $p=.030)$ as well as between T3 and T4 $(F(1,7)=5.854$, $p=.046)$. These early and later effects run in opposite direction, however, and at present, do not appear

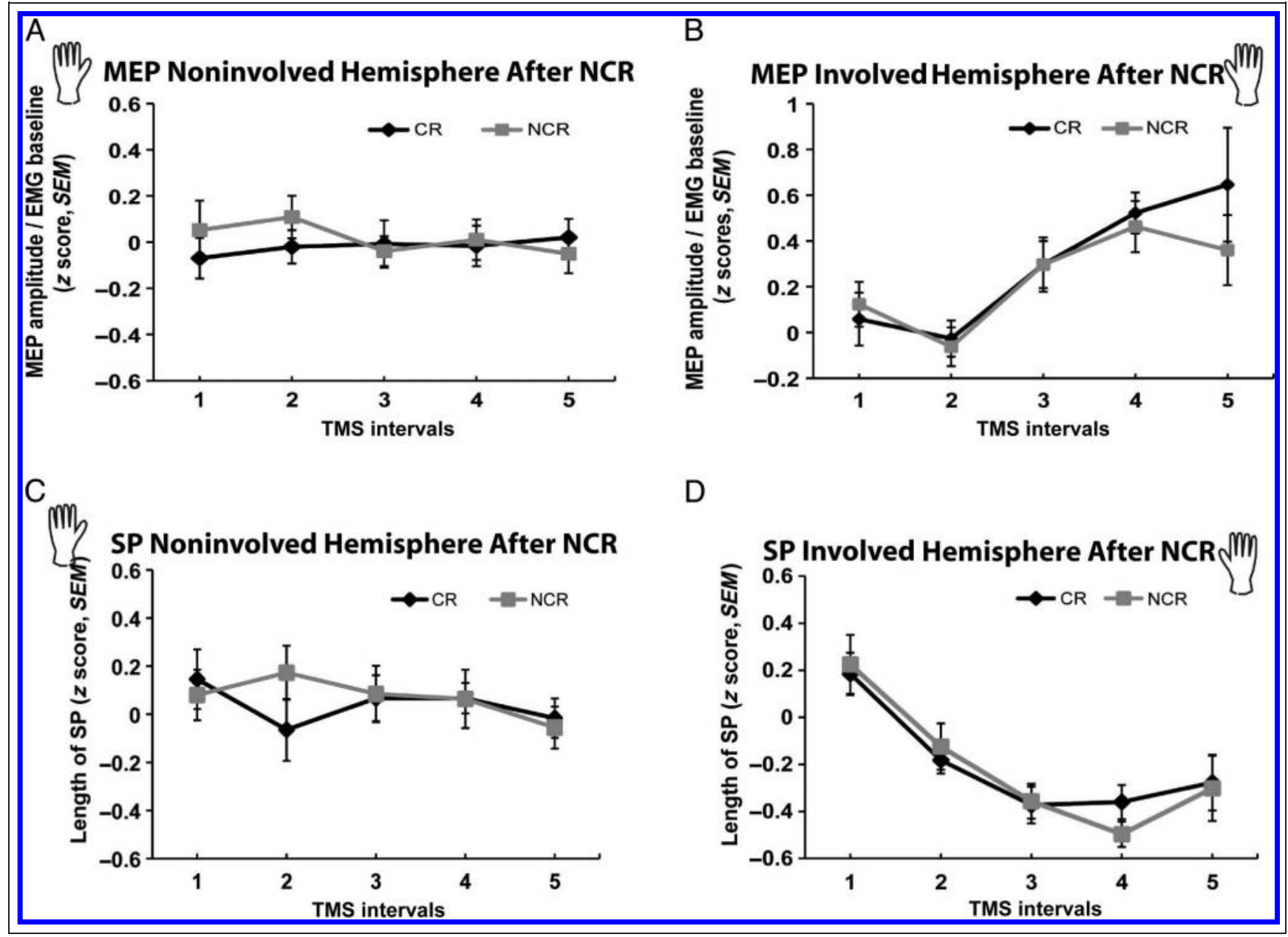

Figure 5. Sequential effects on activation and inhibition dynamics: trials following NCR trials. Details and panel organization are identical to those in Figure 3. 
to reflect a systematic pattern open to straightforward interpretation.

\section{Summary}

Sequential effects modulated the patterns for MEP amplitude and SP duration, in particular, when the stimulated hemisphere was involved in the correct action. Trials preceded by CR trials showed facilitation of the imminent CR responses, but also to hinder disinhibition of imminent NCR responses.

\section{Linking Behavioral and Physiological Data}

Reduced accuracy for fast responses to NCR stimuli has been taken to reflect the expression of potent action impulses incited by the task-irrelevant location of the stimulus. Activation of the incorrect response hand is also thought to be expressed by the amplitude of the MEP for the noninvolved hand at short TMS intervals. Indeed, we observed a strong negative correlation between the accuracy levels of the fastest bin of NCR responses performed with the (right-hand) effector controlled by the stimulated hemisphere and MEP amplitude on NCR trials at time interval T3 when the stimulated hemisphere was involved in the incorrect rather than correct response $(r=-820, p=.004)$. Thus, individuals who make more fast errors on NCR trials also tend to have higher direct activation of the motor cortex controlling the incorrect response as triggered by the NCR stimulus location.

Shortening of SP duration when the stimulated hemisphere was involved in the correct action reflects the disinhibition of the imminent response. Our data showed that NCR responses are disinhibited more slowly (at later TMS intervals) than CR responses. This finding may reflect the fact that the response to NCR stimuli is kept in check until the conflict between competing responses is resolved. For NCR trials at T3, SP duration showed a strong positive correlation with delta values at the fastest quartiles of the RT distribution $(r=.930, p<.001$ for delta value $1 ; r=.868, p=.001$ for delta value 2 ). Thus, individuals who have a large Simon effect in the early segments of the RT distribution, reflecting a need for stronger selective suppression of incorrect action impulses, also show weaker physiological response disinhibition for NCR stimuli.

In summary, the correlation patterns suggest that the expression and (early) suppression of action impulses as reflected by MEP and SP measures correspond directly to behavioral expressions of these processes, as measured through parameters of RT distributions for CR and NCR trials.

\section{DISCUSSION}

The goal of this study was to clarify physiological mechanisms of response capture and suppression of action impulses during conflict at the level of the motor system. We combined single-pulse TMS over M1 with measurements of MEP and SP recorded during a Simon task. MEP amplitude and SP duration changed dynamically over time and differentiated between CR and NCR trials. In the next section, first, the main physiological findings related to action selection, response capture, and selective suppression are discussed in relation to resolving response conflict. Second, we discuss the neural network potentially involved in implementing these processes and the implications of such a network for existing theoretical frameworks.

\section{Action Selection}

The dynamics of the MEP revealed a progressive increase in cortico-spinal excitability before the contralateral overt (correct) response. On CR trials, cortico-spinal excitability increased earlier (i.e., at about $140 \mathrm{msec}$ following stimulus onset) compared with NCR trials, matching the typical behavioral finding in conflict tasks that CR responses are faster than NCR responses. Furthermore, early activation on CR trials was more pronounced when preceded by another CR trial compared with when preceded by an NCR trial. In the latter case, the facilitation effect disappeared altogether. Similar modulations of cortico-spinal excitability have been reported in the context of task switching (Bestmann et al., 2008). Michelet, Duncan, and Cisek (2010) used a Flanker task in which responses were made with either the flexor or the extensor of the same hand. Facilitation of the agonist was accompanied with a decrease in MEP amplitude of the antagonist. Klein-Flügge and Bestmann (2012) replicated these findings in a value-based decision task and showed that the response competition between selected and unselected responses occurred parallel in time with the value-based decision process.

\section{Response Capture}

On conflict trials, the irrelevant stimulus dimension (location) facilitates activation of the hand ipsilateral to the location hence constitutes a source of response conflict. Using TMS to probe the hemisphere not involved in the correct response, we observed early response capture on NCR trials, reflected by increased MEP amplitudes as early as 142 msec after the onset of the visual stimulus. Note that this activation on NCR trials was observed although our analyses were restricted to correct response trials only. This manifestation of early response capture is further supported by the correlation between levels of activation of this hemisphere (when it was associated with the incorrect rather than correct response), as expressed by increased MEP amplitude, and behavioral response capture, as expressed by the high number of fast errors made on NCR trials. This behavioral and physiological pattern suggests that the capture of inappropriate action 
impulses requires suppression to produce correct overt actions.

\section{Selective Suppression}

First, an early selective increase of physiological inhibition on NCR trials suggests active attempts to suppress response capture shown when the stimulated hemisphere was associated with the incorrect action (142 msec). The current study is the first to show this specific physiological inhibition (reflected by SP) that serves to actively suppress incorrect response capture.

Second, the inhibition component of the hemisphere involved in the correct action changes dynamically over time (T1, T2, and T3) and differentiates between CR and NCR trials: Inhibition levels on NCR trials are sustained for a longer period than on CR trials. This study shows that the hemisphere involved in the correct action starts off with high levels of inhibition; these levels decrease over time faster for CR than NCR trials, attaining similar levels of inhibition at T3. No effect of sequence is observed for the SP of the directly involved hemisphere, which dissociates the inhibitory effects from the activation dynamics of the involved hemisphere.

Third, a general decrease of inhibition during late time intervals (T4-T5) suggests that the inappropriate action is disinhibited only at a relatively late stage of the response process. This pattern suggests that responses are kept in check until they no longer constitute a risk for premature overt action. This disinhibition effect may result from the release of lateral inhibition (Meynier, Burle, Possamai, Vidal, \& Hasbroucq, 2009) or from the release of topdown inhibition (Burle, Vidal, Tandonnet, \& Hasbroucq, 2004), in either case implemented presumably through the BG (Ridderinkhof et al., 2011).

\section{Resolving Response Conflict}

During NCR trials, correct responses are disinhibited more slowly (at later TMS intervals) than during CR trials (as evidenced by SP duration for the involved hemisphere for NCR trials at T3). This finding may reflect the fact that the response to NCR stimuli is kept in check until the conflict between competing responses is resolved. This is further supported by the strong positive correlation between SP duration for the involved hemisphere for NCR trials at T3 and delta values at the fastest quartiles of the RT distribution. Thus, individuals who have a large Simon effect in the early segments of the RT distribution reflecting a need for stronger selective suppression of incorrect action impulses also have less physiological response disinhibition for NCR stimuli.

\section{Levels of Inhibition}

Physiological inhibition may occur at various levels, ranging from intrahemispheric inhibition (Carson, 2005) to inhi- bition at the spinal level (Burle et al., 2002, 2004). The SP reflects inhibitory mechanisms at both the spinal level and the cortical level. Spinal inhibition accounts for the first approximately $50 \mathrm{msec}$, and the second part represents the involvement of cortical inhibitory circuits (Chen, Lozano, \& Ashby, 1999; Wilson, Lockwood, Thickbroom, \& Mastaglia, 1993). In general, cortical inhibition reflected by the SP is mediated by $\mathrm{GABA}_{\mathrm{B}}$ receptors. Other inhibitory measures with TMS, for instance, short intracortical inhibition (SICI), are mediated by $\mathrm{GABA}_{\mathrm{A}}$ receptors (Paulus et al., 2008; Di Lazzaro, Oliviero, Pilato, \& Saturno, 2004; Ziemann, Lönnecker, Steinhoff, \& Paulus, 1996). The exact interplay of these different physiological inhibitory mechanisms is still unknown.

Previous research on action programming showed sustained levels of SICI in case of prepared responses and a disappearance of SICI in case of action reprogramming (Neubert, Mars, Olivier, \& Rushworth, 2011). Opposite effects in terms of increased SICI were found in case of pure response inhibition suggesting different underlying mechanisms of inhibition (Coxon, Stinear, \& Byblow, 2006). Duque, Lew, Mazzocchio, Olivier, and Ivry (2010) provided evidence for two dissociable inhibitory mechanisms during response preparation involved in impulse control versus competition resolution. Decreased MEP amplitudes and suppressed H-reflexes at the spinal level, measured before the overt response, suggested an impulsecontrol component. This reduced excitability prevents the premature activation of responses, both at the cortical level and the spinal level, and is only found in case of preselected target muscles. Decreased MEP in the nonselected target muscles suggests a competition-resolution related inhibition to help select the correct response. This competition-resolution related inhibition may either arise from lateral or top-down input (Duque et al., 2010). This notion is further supported by paired-pulse TMS showing a facilitating influence from pre-SMA on M1 and an inhibitory influence from rIFG on M1 (Neubert et al., 2010).

Interestingly, rTMS stimulation over the dorsal premotor cortex, thereby inducing a temporal dysfunction of this area, reduced the impulse-related inhibition suggested to prevent preactivation of selected response. Conversely, rTMS over lateral pFC was associated with decreased inhibition in selecting the appropriate response (Duque, Labruna, Verset, Olivier, \& Ivry, 2012). Future studies may aim to determine with more precision how these different levels of inhibition interact.

\section{Temporal Dynamics}

The use of TMS helped unraveling the time-specific changes in activation and inhibition at the cortico-spinal level that underlie the incidence as well as the resolution of response conflict. The present results, with respect to the temporal dynamics, are in line with previous TMS work on action control. For example, Taylor and colleagues (2007) reported early inhibitory effects during Flanker 
performance as soon as $184 \mathrm{msec}$. Second, in the context of the stop paradigm, excitability of inhibitory interneurons that drive SP prolongation was evident as early as $134 \mathrm{msec}$ following the instruction to stop (van den Wildenberg, Burle, et al., 2010). It should be noted that the effects reported here represent averages across participants. Nevertheless, the timing of TMS was based on individual RT. This individual timing approach ensured that the TMS intervals occurred within the same phase of the response process across participants. However, interindividual differences in RT distribution were relatively small in the early bins but tended to increase for later RT bins. In line with previous literature on this topic (e.g., van den Wildenberg, Burle, et al., 2010; Taylor et al., 2007), our results suggest a dynamic interplay of activation and inhibitory components starting very early in the response process.

Overall, this study highlights the interplay of response capture and selective inhibition processes resulting in dynamic patterns of cortico-spinal excitability within the motor cortex. In the next section, we discuss the neural network potentially involved in implementing these processes in relation to existing theoretical frameworks.

\section{Neural Network Conflict Dynamics, Revisited}

The pre-SMA and rIFC are related to, respectively, selective activation and inhibition of motor responses during the Simon task (Forstmann, Jahfari, et al., 2008; Forstmann, van den Wildenberg, et al., 2008). These findings are in line with the DPAS model, which explains conflict effects and, specifically, the reduction in the conflict effect as a function of RT. Involuntary activation (response capture), because of the location of the stimuli (pre-SMA), is surmised to be followed by active suppression (rIFC) of this undesired response. A similar activation-followed-bysuppression model has been invoked in accounting for conflict patterns in masked-priming tasks (e.g., Eimer \& Schlaghecken, 2003). In this model, inhibition is assumed to be automatic rather than goal directed. Still, other models suggest a more passive decay of response capture over time without an active form of suppression (Hommel, 1993, 1994). Although the present data appear more consistent with an active selective suppression view, the DPAS model and alternative conjectures share the notion of framing the activation dynamics in a time-sequential manner: Response capture is followed by the reduction of this direct activation (either through active selective suppression, automatic inhibition, or passive decay).

However, the present data suggest that response capture and suppression processes may run in parallel rather than in strict sequence. Activation as well as (dis)inhibitory changes occur in ipsilateral as well as contralateral corticospinal systems, commencing already early in the response process and evolving continually over time. Task-irrelevant action impulses capture the incorrect response by producing early activation of the corresponding hemisphere; this capture is countered by inhibitory components at
M1, in this study, already at early stages as well. The typical RT slowing observed during conflict compared with CR trials results from delayed disinhibition of activation of the hemisphere associated with the correct response. The stronger (and/or earlier) the inhibition of the incorrect response, the faster the correct response can be disinhibited.

The BG and parietal regions areas are known to have direct connections to the M1 either via excitatory or inhibitory neurons (Koch et al., 2010; Aravamuthan, Muthusamy, Stein, Aziz, \& Johansen-Berg, 2007; Behrens et al., 2003). The different inhibitory contributions (direct, hyperdirect, and indirect routes; e.g., Aron, Behrens, Smith, Frank, \& Poldrack, 2007; Frank, Samanta, Moustafa, \& Sherman, 2007) of the BG could give rise to the changes in the dynamics of the activation and inhibitory patterns obtained in this study. Recent findings of the contribution of parietal regions in action planning and attention in action interference might relate to the strong facilitation found after repeating CR trials (Brown, Friston, \& Bestmann, 2011; Cui \& Andersen, 2011). This facilitation indeed suggests an attentional bias toward the spatial location of the cue, which could be mediated by parietal-motor connections.

\section{Conclusion}

This study highlights the temporal dynamics of several physiological processes within the cortico-spinal motor system during an action-conflict task. First, the manifestation of response capture and selective suppression of action impulses, and their timing, can be traced at the physiological level. Second, these effects arise early and simultaneously. Third, effects in the hemisphere involved in the incorrect response are paralleled by effects in the hemisphere involved in the correct response. And finally, RT slowing on conflict trials results from delayed disinhibition of the correct response.

\section{Acknowledgments}

This work was supported by an Open Competition grant (K. R. R., W. P. M. v. d. W.) from the Netherlands Organization for Scientific Research (NWO).

Reprint requests should be sent to A. Dilene van Campen, Department of Psychology, University of Amsterdam, Weesperplein 4, 1018 XA Amsterdam, The Netherlands, or via e-mail: A.D. vanCampen@gmail.com.

\section{REFERENCES}

Aravamuthan, B. R., Muthusamy, K. A., Stein, J. F., Aziz, T. Z., \& Johansen-Berg, H. (2007). Topography of cortical and subcortical connections of the human pedunculopontine and subthalamic nuclei. Neuroimage, 37, 694-705.

Aron, A. R., Behrens, T. E., Smith, S., Frank, M. J., \& Poldrack, R. A. (2007). Triangulating a cognitive control network using diffusion-weighted magnetic resonance imaging (MRI) and functional MRI. The Iournal of Neuroscience, 27, 3743-3752.

Behrens, T. E. J., Johansen-Berg, H., Woolrich, M. W., Smith, S. M., Wheeler-Kingshott, C. A. M., Boulby, P. A., et al. (2003). 
Non-invasive mapping of connections between human thalamus and cortex using diffusion imaging. Nature Neuroscience, 6, 750-757.

Bestmann, S., Harrison, L. M., Blankenburg, F., Mars, R. B., Haggard, P., Friston, K. J., et al. (2008). Influence of uncertainty and surprise on human corticospinal excitability during preparation for action. Current Biology, 18, 775-780.

Brown, H., Friston, K., \& Bestmann, S. (2011). Active inference, attention, and motor preparation. Frontiers in Psychology, 2, 218.

Burle, B., Bonnet, M., Vidal, F., Possamaï, C.-A., \& Hasbroucq, T. (2002). A transcranial magnetic stimulation study of information processing in the motor cortex: Relationship between the silent period and the reaction time delay. Psvchopbusiology, 39, 207-217.

Burle, B., Vidal, F., Tandonnet, C., \& Hasbroucq, T. (2004). Physiological evidence for response inhibition in choice reaction time tasks. Brain and Cognition, 56, 153-164.

Carson, R. G. (2005). Neural pathways mediating bilateral interactions between the upper limbs. Brain Research Reviews, 49, 641-662.

Chen, R., Lozano, A. M., \& Ashby, P. (1999). Mechanism of the silent period following transcranial magnetic stimulation. Experimental Brain Research, 128, 539-542.

Coxon, J. P., Stinear, C. M., \& Byblow, W. D. (2006). Intracortical inhibition during volitional inhibition of prepared action. Lournal of Neuropbysiology, 95, 3371-3383.

Cui, H., \& Andersen, R. A. (2011). Different representations of potential and selected motor plans by distinct parietal areas. The Iournal of Neuroscience, 31, 18130-18136.

Di Lazzaro, V., Oliviero, A., Pilato, F., \& Saturno, E. (2004). The physiological basis of transcranial motor cortex stimulation in conscious humans. Clinical Neuropbysiology, 115, $255-266$

Duque, J., Labruna, L., Verset, S., Olivier, E., \& Ivry, R. B. (2012). Dissociating the role of prefrontal and premotor cortices in controlling inhibitory mechanisms during motor preparation. The Iournal of Neuroscience, 32, 806-816.

Duque, J., Lew, D., Mazzocchio, R., Olivier, E., \& Ivry, R. B. (2010). Evidence for two concurrent inhibitory mechanisms during response preparation. The Iournal of Neuroscience, 30, 3793-3802.

Egner, T. (2008). Multiple conflict-driven control mechanisms in the human brain. Trends in Cognitive Sciences, 12, 374-380.

Egner, T., \& Hirsch, J. (2005). Cognitive control mechanisms resolve conflict through cortical amplification of task-relevant information. Nature Neuroscience, 8, 1784-1790.

Eimer, M., \& Schlaghecken, F. (2003). Response facilitation and inhibition in subliminal priming. Biological Psychology 64, 7-26

Eriksen, B. A., \& Eriksen, C. W. (1974). Effects of noise letters upon the identification of a target letter in a nonsearch task. Perception \& Psychopbysics, 16, 143-149.

Forstmann, B. U., Jahfari, S., Scholte, H. S., Wolfensteller, U., van den Wildenberg, W. P. M., \& Ridderinkhof, K. R. (2008). Function and structure of the right inferior frontal cortex predict individual differences in response inhibition: A model-based approach. The Iournal of Neuroscience, 28, 9790-9796.

Forstmann, B. U., van den Wildenberg, W. P. M., \& Ridderinkhof, K. R. (2008). Neural mechanisms, temporal dynamics, and individual differences in interference control. Lournal of Cognitive Neuroscience, 20, 1854-1865.

Frank, M. J., Samanta, J., Moustafa, A. A., \& Sherman, S. J. (2007). Hold your horses: Impulsivity, deep brain stimulation, and medication in Parkinsonism. Science, 318, 1309-1312.

Hasbroucq, T., Kaneko, H., Akamatsu, M., \& Possamaï, C.-A. (1997). Preparatory inhibition of cortico-spinal excitability: A transcranial magnetic stimulation study in man. Cognitive Brain Research, 5, 185-192.

Hommel, B. (1993). Inverting the Simon effect by intention. Psvchological Research, 55, 270-279.

Hommel, B. (1994). Effects of irrelevant spatial S-R compatibility depends on stimulus complexity. Psvchological Research, 56, 179-184.

Klein-Flügge, M. C., \& Bestmann, S. (2012). Time-dependent changes in human corticospinal excitability reveal value-based competition for action during decision processing. The Iournal of Neuroscience, 32, 8373-8382.

Koch, G., Cercignani, M., Pecchioli, C., Versace, V., Oliveri, M., Caltagirone, C., et al. (2010). In vivo definition of parieto-motor connections involved in planning of grasping movements. Neuroimage, 51, 300-312.

Kornblum, S., Hasbroucq, T., \& Osman, A. (1990). Dimensional overlap: Cognitive basis for stimulus-response compatibilityA model and taxonomy. Psvchological Review, 97, 253.

MacLeod, C. M. (1991). Half a century of research on the Stroop effect: An integrative review. Psychological Bulletin, 109, $163-203$

Meynier, C., Burle, B., Possamaï, C.-A., Vidal, F., \& Hasbroucq, T. (2009). Neural inhibition and interhemispheric connections in two-choice reaction time: A Laplacian ERP study. Psychophysiology, 46, 726-730.

Michelet, T., Duncan, G. H., \& Cisek, P. (2010). Response competition in the primary motor cortex: Cortico-spinal excitability reflects response replacement during simple decisions. Journal of Neurophysiologv 104, $119-127$

Neubert, F.-X., Mars, R. B., Buch, E. R., Olivier, E., \& Rushworth, M. F. S. (2010). Cortical and subcortical interactions during action reprogramming and their related white matter pathways. Proceedings of the National Academv of Sciences. U.S.A., 107, 13240-13245.

Neubert, F.-X., Mars, R. B., Olivier, E., \& Rushworth, M. F. S. (2011). Modulation of short intra-cortical inhibition during action reprogramming. Experimental Brain Research. 211, 265-276.

Paulus, W., Classen, J., Cohen, L. G., Large, C. H., Di Lazzaro, V., Nitsche, M., et al. (2008). State of the art: Pharmacologic effects on cortical excitability measures tested by transcranial magnetic stimulation. Brain Stimulation, 1, 151-163.

Ridderinkhof, K. R. (2002). Micro- and macro-adjustments of task set: Activation and suppression in conflict tasks. Psychological Research, 66, 312-323.

Ridderinkhof, K. R., Forstmann, B. U., Wylie, S. A., Burle, B., \& van den Wildenberg, W. P. M. (2011). Neurocognitive mechanisms of action control: Resisting the call of the sirens. Wilev Interdisciplinary Reviews: Cognitive Science, 2, 174-192.

Rossi, S., Hallett, M., Rossini, P. M., \& Pascual-Leone, A. (2009). Safety, ethical considerations, and application guidelines for the use of transcranial magnetic stimulation in clinical practice and research. Clinical Neuropbysiology, 120, 2008-2039.

Simon, J. R. (1969). Reactions toward the source of stimulation. Lournal of Experimental Psychology, 81, 174-176.

Stürmer, B., Siggelkow, S., Dengler, R., \& Leuthold, H. (2000). Response priming in the Simon paradigm. A transcranial magnetic stimulation study. Experimental Brain Research, 135, 353-359.

Taylor, P. C. J., Nobre, A. C., \& Rushworth, M. F. S. (2007). Subsecond changes in top down control exerted by human medial frontal cortex during conflict and action selection: A combined transcranial magnetic stimulation electroencephalography study. The Iournal of Neuroscience, 27, 11343-11353. 
Terao, Y., \& Ugawa, Y. (2002). Basic mechanisms of TMS. Iournal of Clinical Neuropbvsiologv, 19, 322-343.

van den Wildenberg, W. P. M., Burle, B., Vidal, F., van der Molen, M. W., Ridderinkhof, K. R., \& Hasbroucq, T. (2010). Mechanisms and dynamics of cortical motor inhibition in the stop-signal paradigm: A TMS study. Journal of Cognitive Neuroscience, 22, 225-239.

van den Wildenberg, W. P. M., Wylie, S. A., Forstmann, B. U., Burle, B., Hasbroucq, T., \& Ridderinkhof, K. R. (2010). To head or to heed? Beyond the surface of selective action inhibition: A review. Frontiers in Human Neuroscience. 4, 222.

Wilson, S. A., Lockwood, R. J., Thickbroom, G. W., \& Mastaglia, F. L. (1993). The muscle silent period following transcranial magnetic cortical stimulation. Lournal of the Neurological Sciences, 114, 216-222.

Ziemann, U., Lönnecker, S., Steinhoff, B. J., \& Paulus, W. (1996). Effects of antiepileptic drugs on motor cortex excitability in humans: A transcranial magnetic stimulation study. Annals of Neurology, 40, 367-378. 


\section{This article has been cited by:}

1. Julie Duque, Ludovica Labruna, Christian Cazares, Richard B. Ivry. 2014. Dissociating the influence of response selection and task anticipation on corticospinal suppression during response preparation. Neuropsychologia 65, 287-296. [CrossRef]

2. Tamar R. Makin, Claudio Brozzoli, Lucilla Cardinali, Nicholas P. Holmes, Alessandro Farnè. 2014. Left or right? Rapid visuomotor coding of hand laterality during motor decisions. Cortex . [CrossRef]

3. Zeshu Shao, Ardi Roelofs, Daniel J. Acheson, Antje S. Meyer. 2014. Electrophysiological evidence that inhibition supports lexical selection in picture naming. Brain Research 1586, 130-142. [CrossRef]

4. Jasper Winkel, Max C. Keuken, Leendert van Maanen, Eric-Jan Wagenmakers, Birte U. Forstmann. 2014. Early evidence affects later decisions: Why evidence accumulation is required to explain response time data. Psychonomic Bulletin \& Review 21, 777-784. [CrossRef] 